\title{
Compuestos organoclorados residuales en dos especies ícticas de la Ciénaga Grande del Bajo Sinú, Córdoba, Colombia
}

\author{
Residual organochlorinated compounds in two fish species from \\ Ciénaga Grande del Bajo Sinú, Córdoba, Colombia
}

Edineldo Lans C, ${ }^{1 *}$ M.Sc, Basilio Díaz $\mathrm{P}^{1}$ Qco. Manuel Paez $\mathrm{M},{ }^{1} \mathrm{Ph} . \mathrm{D}$.

1 Universidad de Córdoba, Facultad de Ciencias Básicas e ingenierías, Instituto Regional del Agua (IRAGUA) Grupo de Investigación en Aguas Pesticidas y Metales Pesados "GIAMP", Montería-Colombia. *Correspondencia: edlans@sinu.unicordoba.edu.co

Recibido: Septiembre de 2009; Aceptado: Julio de 2010.

\section{RESUMEN}

Objetivo. Determinación de niveles de pesticidas organoclorados (POCs) en especies ícticas, procedentes de la Ciénaga Grande del Bajo Sinú, Materiales y métodos. Se usó un cromatógrafo de gases (Perkin Elmer Autosystem $\mathrm{XL}$ ) con detector captura de electrones y columna capilar $5 \%$ de difenil y $95 \%$ de dimetilpolisiloxano, de $30 \mathrm{~m}, 0.25 \mathrm{~mm}$ de diámetro interno y $0.25 \mu \mathrm{m}$ de espesor de película. Las especies estudiadas fueron: bocachico (Prochilodus magdalenae) y moncholo (Hoplias malabaricus), durante las estaciones seca y húmeda. Resultados. El porcentaje de recuperación de los pesticidas determinados se registró entre 80 y $84 \%$, mientras que los límites de detección y cuanficación fueron 0.02 a $0.18 \mathrm{ng} / \mathrm{g}$ y 0.2 a $1.8 \mathrm{ng} / \mathrm{g}$ respectivamente. La desviación estándar relativa permaneció por debajo del $7 \%$ en todos los casos. De los pesticidas organoclorados investigados, en las dos especies se detectaron el $\alpha$-clordano, endrín y aldrín. Se observó que a medida que aumenta el peso y el tamaño del pez, aumenta la concentración de los plaguicidas en una de las especies. Los niveles de concentración de los POCs estuvieron en el intervalo de $0.35-3.75$ y $0.48-5.49 \mathrm{ng} / \mathrm{g}$ para el moncholo (Hoplias malabaricus) and bocachico (Prochilodus magdalenae) respectivamente. Conclusiones. La frecuencia de aparición de POCs y los niveles detectados en las especies podrían ser consecuencia de actividades antropogénicas realizadas en los alrededores de la ciénaga. La correlación entre las medidas biométricas y la concentración depende de la especie íctica. Las concentraciones encontradas en las especies estudiadas, Hoplias malabaricus y Prochilodus magdalenae no representan toxicidad letal inmediata.

Palabras clave: Peces, análisis, pesticidas, toxicidad. (Fuente: AIMS) 


\section{ABSTRACT}

Objective: To determine the levels of organochlorinated pesticides (OCPs) in two fish species, obtained from Ciénaga Grande del Bajo Sinu. Materials and methods. A gas chromatograph (Perkin Elmer XL Autosystem) with electron capture detector and capillary column $5 \%$ diphenyl and $95 \%$ dimethylpolysiloxane, with $30 \mathrm{~m}$ in length, $0.25 \mathrm{~mm}$ internal diameter and $0.25 \mathrm{~mm}$ thick film were used. Two species were studied: Prochilodus magdalenae and Hoplias malabaricus during wet and dry seasons. Results. The percentages of pesticides recovered were 80 and $84 \%$ whereas the detection and quantification limit was 0.02 to $0.18 \mathrm{ng} / \mathrm{g}$ and 0.2 to $1.8 \mathrm{ng} / \mathrm{g}$ for both species, respectively. Relative standar deviation remained below $7 \%$ in all cases. Among those organochlorinated investigated here, $\alpha$-clordane, endrín and aldrín were detected in both fish species. In one of the species studies, it was observed that when weight and size of fishes incresed the pesticides concentration also increased. The concentration levels of OCPs were in the range of 0.35-3.75 and 0.48-5.49 $\mathrm{ng} / \mathrm{g}$ for moncholo (Hoplias malabaricus) and bocachico (Prochilodus magdalenae) respectively. Conclusions. The frequency of OCPs and the levels detected in these species might be the result of anthropogenic activities conducted in the vicinity of the lake. The correlation between the biometric measurements and concentration was depending on the fish species. The concentrations found in the studied species Hoplias malabaricus and Prochilodus magdalenae do not represent an immediately lethal toxicity.

Key words: Fishes, analysis, pesticides, toxicity. (Source: AIMS)

\section{INTRODUCCIÓN}

La Ciénaga Grande del Bajo Sinú está ubicada al norte del departamento de Córdoba, circunscrita por los municipios de Lorica, Purísima, Momil, Ciénaga de Oro, San Pelayo y Chimá. (Figura 1). Consta de 42.000 hectáreas aproximadamente de los cuales 18.000 hectáreas pertenecen al municipio de Lorica tiene una profundidad en época lluviosa que oscila entre 4.5 y 5.5 metros (1). La Ciénaga ha sido la principal despensa durante muchas generaciones para sus comunidades, como riqueza natural ha proporcionado por muchas décadas alimento y bienestar a muchas poblaciones ribereñas que viven de ella (2).

La Ciénaga ha sido una despensa agrícola de muchas especies como el prochilodus magdalenae, hoplias malabaricus y otras especies menores.

Los plaguicidas organoclorados (POCs) hacen parte de la lista de Contaminantes Orgánicos Persistentes (COP), identificados por el Programa para el Ambiente de Naciones Unidas (UNEP), por sus efectos sobre la biota acuática y terrestre, así como sobre la salud pública (3).
Los POCs son generalmente encontrados en mezclas complejas y su presencia es una indicación de contaminación antropogénica. Aunque su producción y comercialización han sido prohibidas en muchos países desde el 1970, existen evidencias de su presencia en muchos ecosistemas acuáticos Colombianos (4).

Una vez liberados al ecosistema lacustre, estos contaminantes se biomagnifican en la cadena alimenticia y a medida que aumenta el nivel trófico en la cadena alimenticia aumentan los niveles de concentración principalmente en el los tejidos grasos $(5,6)$.

Los POCs se absorben y después se redistribuyen a los tejidos con elevado carácter lipofílico. Pero de todos los tejidos del pez, la medición de los plaguicidas en el músculo reviste mayor importancia porque, allí se establece un indicador de contaminación que aporta información sobre los efectos en la salud de los consumidores (7).

El bocachico que pertenece al genero prochilodus es una especie iliófaga que 


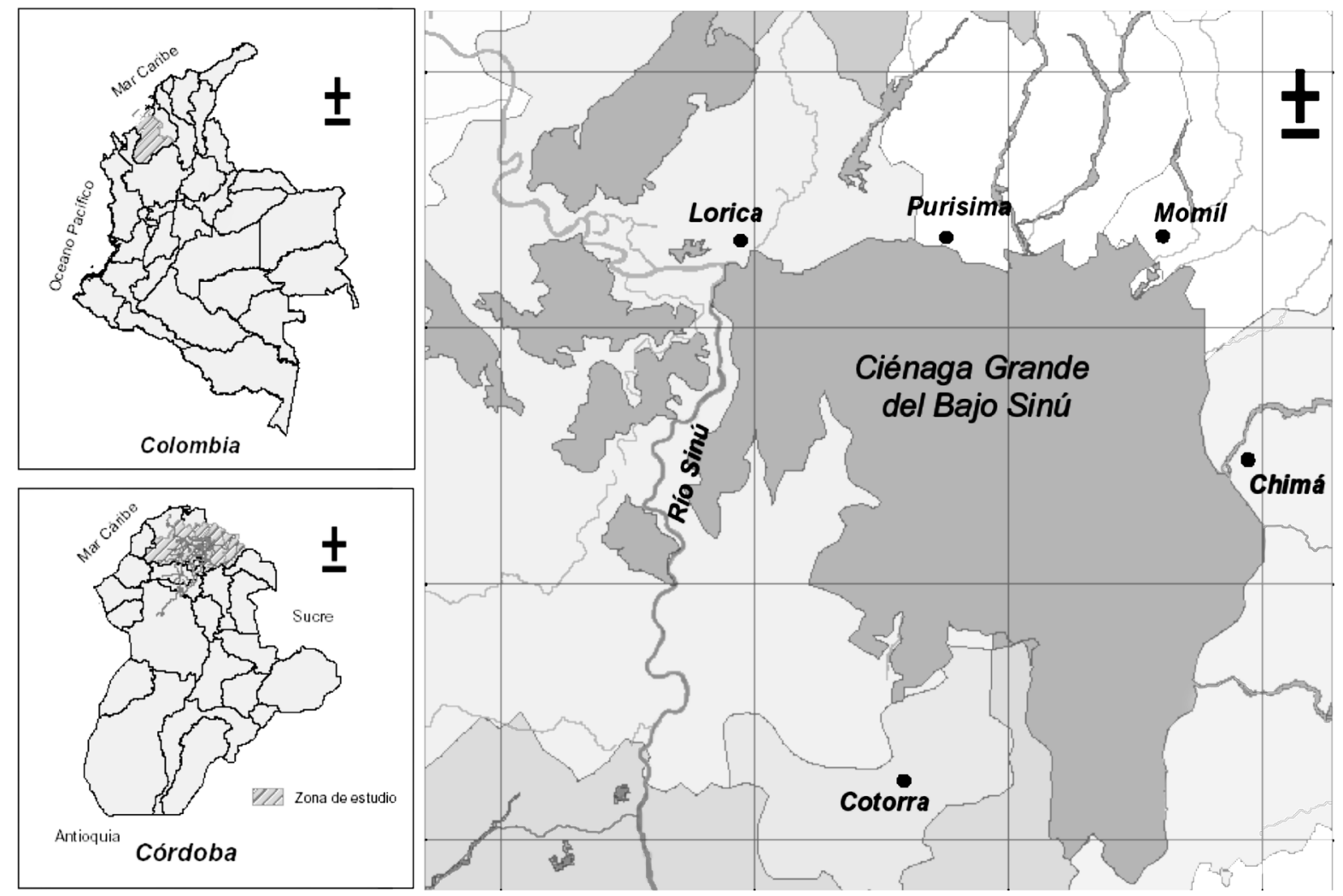

Figura 1. Ciénaga Grande del Bajo Sinú.

habita en aguas dulces, especialmente en los grandes ríos y los sistemas lacustres sudamericanos, y sirve como fuente de alimentación convirtiéndose, en algunas ocasiones en única fuente de subsistencia (8).

Otra especie muy comercial que hace parte de la alimentación de pobladores que habitan los alrededores de las ciénagas es el Moncholo (Hoplias malabaricus), de amplia distribución en América del Sur, siendo encontrado en casi todas las aguas lénticas y lóticas (9). Es carnívoro y voraz, en sus estados iniciales es zooplanctófago, luego entomófago (consume insectos) y después se convierte en ictiófago consumiendo especies pequeñas como sardinas, Astyanax sp; mojarritas, Aequidens sp y viejitas, Curimata sp. (10). Sin embargo, no hay estudios que describan los niveles de pesticidas organoclorados en el tejido de las especies icticas Moncholo y Bocachico, presentes en la Ciénaga Grande del Bajo Sinú y de alto consumo por parte de los pobladores de la región (11).
La exposición de los seres humanos a los COP constituye una preocupación seria debido a los efectos negativos que incluyen defectos de nacimiento, disrupción endocrina y problemas reproductivos $(12,13)$. Es por ello que el presente estudio pretende determinar los niveles de pesticidas organoclorados en especies ícticas, en la Ciénaga Grande del Bajo Sinú con el objeto de alertar sobre sus efectos en la salud publica y riesgos sobre este ecosistema.

\section{MATERIALES Y MÉTODOS}

Se realizaron seis muestreos durante un año cubriendo los picos climáticos húmedo, seco y de transición o lluvias menores de la zona. Se seleccionaron dos especies ícticas; Bocachico (prochilodus magdalenae) y Moncholo (hoplias malabaricus), 11 unidades por especie teniendo en cuenta su importancia comercial y su relación trófica con el ecosistema. Las muestras de peces 
se compraron a los pescadores de la zona. Los peces recolectados fueron pesados y medios. (Tabla 2)

Los solventes (acetonitrilo, hexano) utilizados para el análisis fueron grado cromatografico (Merck, con $99.5 \%$ de pureza).

Para el análisis de la muestra se pesó $0.5 \mathrm{~g}$ (en base húmeda) de tejido muscular. Dicha masa fue macerada y homogenizada para posteriores tratamientos. La extracción de los POC se llevó a cabo utilizando $2 \mathrm{~mL}$ de acetonitrilo y posterior centrifugación durante $10 \mathrm{~min}$ a $90 \mathrm{rpm}$; repitiendo el procedimiento dos veces. El sobrenadante luego es recolectado. Al sobrenadante se le agregan $25 \mathrm{~mL}$ de solución acuosa de sulfato de sodio( $2 \% \mathrm{p} / \mathrm{v})$, se agita y se deja en reposo por $10 \mathrm{~min}$. La mezcla de acetonitrilo acuoso se extrae con dos porciones de 5 y $2 \mathrm{~mL}$ de hexano, el extracto es concentrado a 0.5 $\mathrm{mL}$ en un rotaevaporador a $45^{\circ} \mathrm{C}, 56 \mathrm{rpm}$ y 0.6 bar de presión.

Los análisis se llevaron a cabo en un cromatógrafo de gases (Perkin Elmer Autosystem $\mathrm{XL}$ ) equipado con un ECD Ni63 (detector captura de electrones) y conectado a un PC con un software Turbochrom Navigator para la adquisición de datos y el control de las condiciones instrumentales. Se usó nitrógeno de alta pureza como gas de arrastre y make up a una velocidad de flujo de $6.2 \mathrm{~mL} / \mathrm{min}$ y fue controlado usando un flujo programado. La columna capilar utilizada fue de $30 \mathrm{~m}$ x $0.25 \mathrm{~mm}$ de diámetro interno y $0.25 \mu \mathrm{m}$ de espesor de pelicula recubierta con $5 \%$ difenil y $95 \%$ de dimetilpolisiloxano de Supelco. La temperatura del inyector y detector fueron de 200 y $250^{\circ} \mathrm{C}$ respectivamente, la del horno fue programada de la siguiente manera: temperatura inicial de 100 oC, a $15^{\circ} \mathrm{C} / \mathrm{min}$ hasta $160^{\circ} \mathrm{C}$ (5 min), 3oC/min hasta $250^{\circ} \mathrm{C}$, temperatura final del análisis. Modo de inyección: splitless. (14)

Para establecer las diferencias estadísticamente significativas entre la concentración de los POCs y las medidas biométricas, la concentración POCs y especies, concentración POCs y periodo estacional se utilizó un análisis de varianza ANOVA $(p<0.05)$ utilizando STATGRAPHICS Plus 2.0

\section{RESULTADOS}

El control de calidad incluyó la calibración del instrumento usando estándares certificados (mezcla de pesticidas marca Supelco) y análisis de muestras dopadas. El coeficiente de determinación del estándar certificado se mantuvo por encima de 0.99. Un estándar fue analizado cada 12 muestras para verificar la calibración del instrumento. El límite de detección de los pesticidas determinados permaneció en el intervalo de 0.02 a $0.18 \mathrm{ng} / \mathrm{g}$. En los pesticidas determinados la DER (desviación estándar relativa) estuvo por debajo del $7 \%$ en todos los casos y el porcentaje de recuperación fue del 80 al $84 \%$ (15).

Los niveles de concentración del aldrín, endrín y a-clordano de algunos peces estuvieron por debajo del límite de detección, para la discusión solo se tuvo en cuenta aquellas concentraciones que fueron iguales o superiores del nivel mínimo detectable (Tabla 1).

Tabla 1. Concentración de cada uno de los POCs hallados en ( $\mathrm{ng} / \mathrm{g}$-peso húmedo) por especie analizada de la Ciénaga Grande del Bajo Sinú

\begin{tabular}{|c|c|c|c|c|c|c|c|c|}
\hline \multirow{2}{*}{ Especies } & \multicolumn{2}{|c|}{ Aldwin } & \multicolumn{2}{|c|}{ Endin' } & \multicolumn{2}{|c|}{ d-Clordano } & \multicolumn{2}{|c|}{ [OCs } \\
\hline & I & p & I & & I & $p$ & 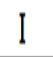 & $p$ \\
\hline Hoplas malabaricus & \multicolumn{8}{|c|}{ 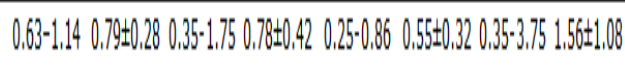 } \\
\hline \multicolumn{9}{|c|}{ 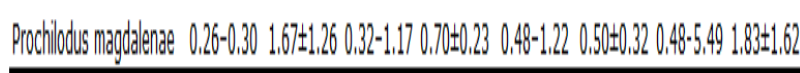 } \\
\hline
\end{tabular}

$\mathrm{I}=$ intervalo de lectura; $\mathrm{P}=$ Valor promedio

A cada una de las muestras de tejido de pez se le estableció las medidas de longitud y peso, antes de realizar el análisis. (Tabla 2).

Con el propósito de evaluar el grado de contaminación debida a POCs en los dos periodos climáticos de la región, se evaluaron muestras en diferentes épocas (Tabla 3). 
Tabla 2. Concentración total de POCs (ng/gpeso húmedo) y medidas biométricas de dos especies ícticas.

\begin{tabular}{|c|c|c|c|c|c|c|}
\hline \multirow{2}{*}{ Especies } & \multicolumn{2}{|c|}{ Longitud(cm) } & \multicolumn{2}{|c|}{$\operatorname{Peso}(g)$} & \multicolumn{2}{|c|}{$\mathrm{ng} / \mathrm{g}$} \\
\hline & I & $p$ & I & T & I & p \\
\hline alias malabal & \multicolumn{6}{|c|}{$22 \cdot 2725 \pm 2118 \cdot 209157 \pm 300.35 \cdot 3.751 .56 \pm 1.00$} \\
\hline \multicolumn{7}{|c|}{ Prochllodus magdalenae $26 \cdot 3529 \pm 2253-462360 \pm 630.48 \cdot 5.491 .83 \pm 1,62$} \\
\hline
\end{tabular}

$\mathrm{I}=$ intervalo de lectura; $\mathrm{P}=$ Valor promedio

Tabla 3. Concentración POCs total en ( $\mathrm{ng} / \mathrm{g}$ peso húmedo) por especies y periodo estacional.

\begin{tabular}{|c|c|c|c|c|}
\hline \multirow{2}{*}{ Especies } & \multicolumn{2}{|c|}{ Época Seca } & \multicolumn{2}{|c|}{ Época Húmedo } \\
\hline & I & P & I & p \\
\hline inlias malabaricus & \multicolumn{4}{|c|}{$0.35 \cdot 3.681 .58 \pm 1.080 .35 \cdot 3.751 .53 \pm 1.17$} \\
\hline \multicolumn{5}{|c|}{ Prochilodus magdalenae $0.48 \cdot 5,421,80 \pm 1,660,52 \cdot 5,491,86 \pm 1,70$} \\
\hline
\end{tabular}

$\mathrm{I}=$ intervalo de lectura; $\mathrm{P}=$ Valor promedio

\section{DISCUSIÓN}

Los niveles en concentración de POCs (Tabla 1) para las especies estudiadas son significativamente diferentes en ambas especies (ANOVA: $d f=14, F=3524$, $\mathrm{P}=0.0130$ ).

La mayor concentración de POCs en el bocachico se debe a la biomagnificación de estas sustancias una vez llegan a los ecosistemas acuáticos y al régimen alimenticio preferido por la especie (iliófaga).

Plata et al (16) determinaron valores de magnificación hasta de 22000 veces en especies carnívoras (Ariopsis bonillai), detritívoras y plactófagas. A pesar de su carácter iliófago en el bocachico no se detectaron niveles de heptacloro epóxido, alfa- hexaclorociclohexano $(\mathrm{a}-\mathrm{HCH})$, betahexaclorociclohexano $(\beta-\mathrm{HCH})$, gammahexaclorociclohexano $(\mathrm{\gamma}-\mathrm{HCH})$ detectado en concentraciones de hasta $4.81 \mathrm{ng} / \mathrm{g}$ en sedimentos de la Ciénaga.(14) Esto es debido a características de metabolismo propias de cada especie para asimilar más eficientemente unos pesticidas, que otros. (17).

Si se tiene en cuenta lo anterior no se explica por que los POCs detectados en sedimentos no se detectaron al menos en el bocachico, conocido su carácter iliófago, por lo que es preciso realizar estudios en este campo. (19) Por su parte la presencia de estos POCs en el moncholo (hoplias malabaricus) se explica por su naturaleza carnívora. (11)

Asimismo, se muestra la concentración de POCs y las medidas biométricas de los peces estudiados en la tabla 2. Existe una diferencia estadísticamente significativa entre la longitud y la concentración de POCs en el bocachico, y entre el peso y la concentración (ANOVA: $\mathrm{df}=6$; $\mathrm{F}=12.45, \quad \mathrm{P}=0.0007 ; \quad \mathrm{df}=15, \quad \mathrm{P}=0.005$, respectivamente), indicando que la concentración de pesticidas detectados aumenta a medida que aumenta la longitud y el peso en esta especie. Mientras que para el moncholo no existe diferencia estadísticamente significativa entre la concentración y el peso, pero sí entre la concentración y la longitud. (ANOVA: $d f=12, \quad F=18.52, \quad P=0.1774$ y $d f=5$, $\mathrm{F}=5.86, \quad \mathrm{P}=0.0087$ respectivamente). Por lo anterior podemos afirmar que las relaciones positivas entre las medidas biométricas y la concentración de POCs dependen de la especie en estudio.

Existen estudios que defienden correlaciones positivas entre el tamaño del pez (longitud) y la concentración de plaguicidas (20). Sin embargo, en esos estudios se suelen comparar grupos (peces de $0-10 \mathrm{~cm}$, peces de $10-20 \mathrm{~cm}$ ) con niveles de plaguicidas (21). En nuestro estudio no se tomaron en cuenta estos intervalos de longitud, pero igualmente se mostró una correlación entre las medidas biométricas y la concentración para las especies con diferencia apreciable en su longitud, como fue el caso del Prochilodus magdalenae.

Giouranovits Psyllidou et al (22), encontraron que la concentración de varios organoclorados (DDT, $\mathrm{HCH}$, aldrín y dieldrín) tiende a incrementarse a medida 
que aumenta el tamaño de los peces, pero no encontraron una correlación consistente. Al respecto se encontró buena correlación respecto al Prochilodus magdalenae, mas no para el hoplias malabaricus. Algunos autores no encuentran relación alguna $(23,24)$, sin embargo; otros determinaron concentraciones de 1.1 dicloro 2.2-bis(p-clorofenil) etileno (DDE) y las correlacionaron con el peso y longitud de las especies capturadas, no encontrando correlación lineal $\left(r^{2}=0.55\right.$ y $r^{2}=0.24$ para el peso y longitud respectivamente) (25). Para estos autores, estos niveles no superan los niveles máximos recomendados en el consumo humano, establecidos por la Organización para la Agricultura y la alimentación (FAO) $(26,27)$.

Las concentraciones letales son cientos de veces más altas que las encontradas en las especies estudiadas, (Hoplias malabaricus y Prochilodus magdalenae) por lo que no representan toxicidad letal inmediata (28). Sustancias como el aldrín, uno de los organoclorados más tóxicos, exhibe concentraciones letales para peces como la trucha arcoiris (Oncorhynchusmykiss) - la perca azul (Micropterus salmoides) de 10 y $8 \mu^{-1} \mathrm{~L}^{-1}$ respectivamente; mientras que el DDT tiene un efecto tóxico menor requiriendo concentraciones entre 8-100 $\mathrm{mg} \mathrm{L}^{-1}$ para provocar la muerte de peces en 96 horas (29).

Si bien las concentración de pesticidas organoclorados detectados en estas especies no son "altas" como para provocar la muerte de organismos, si es un factor negativo en este ecosistema, porque aún niveles más bajos pueden conducir simultáneamente a otros problemas ecológicos a largo plazo, como son los asociados a procesos de bioacumulación y biomagnificación a través de la cadena trófica (30).

Los niveles en concentración de POCs para ambas especies por periodo estacional se muestran en la tabla 3. Concentraciones altas se encuentran tanto en el periodo húmedo como en el seco para el bocachico, estableciéndose además una diferencia estadísticamente significativa entre períodos estacionales en ambas especies (ANOVA: $p<0.05$ ). Se puede afirmar entonces que el período estacional tiene influencia en los niveles de concentración de POCs teniendo en cuenta la especie. Se observa que dichas diferencias son aún mas marcadas en el período húmedo para el bocachico, por ser la época en la cual ocurren los mayores aportes como consecuencia de las corrientes de aguas provenientes de las tierras de cultivo y al carácter iliófago de éste. En el moncholo se observa un aumento de la concentración de los POCs en el período seco, contrastando con el bocachico.

Del total de muestras analizadas durante la época de estudio, la frecuencia de aparición del a-clordano y el endrín en ambas especies fué de un $73 \%$, en tanto que para el aldrín esta frecuencia fué de $18 \%$ y $27 \%$ para el moncholo y bocachico respectivamente. Para el bocachico el aldrín se encontró en un 33\% más que en el moncholo, mientras que, con respecto al endrín y el a-clordano, el aldrín se encontró en un 63 y $75 \%$ más en bocachico que en moncholo. La alta frecuencia de aparición del endrin y el a-clordano, indica el mayor uso de agroquímicos con contenido de estos pesticidas.

La frecuencia de aparición de POCs, así como los niveles detectados en las especies estudiadas, permiten correlacionar dicho fenómeno con actividades antropogénicas realizadas en los alrededores de la ciénaga, posiblemente como consecuencia de actividades agrícolas. Estos agroquímicos, no obstante de estar prohibidos debido a sus efectos adversos a la salud, actualmente están siendo utilizados, contribuyendo al proceso de biomagnificación de estas sustancias e incrementando el riesgo por exposición a la población, debido al consumo masivo de estas especies ya que hace parte de su dieta alimentaria diaria.

Finalmente, no se conoce completamente a que se debe que algunas especies ícticas absorban más una clase de pesticidas que otras, por lo que es necesario realizar estudios tendientes a identificar dichas características. 


\section{REFERENCIAS}

1. CVS, U.de A. Estudio ecológico y ambiental de la Ciénaga Grande de Lorica. Montería: CVS.1993.

2. CVS, Estudios básicos para un plan de ocupación del espacio en la cuenca del rio Sinú. Informe. Montería: Corporación Autónoma de los Valles del Sinu y San Jorge CVS.1985.

3. UNEP: Draft Guidance on the Global Monitoring Plan for Persistent Organic Pollutants. UNEP, February 2007

4. Lans E, Marrugo J, Díaz B. Estudio de la Contaminación por Pesticidas Organoclorados en Aguas de la Cienaga Grande del Bajo Sinú, Córdoba. Colombia. Temas Agrarios. Universidad de Córdoba, 2008; 13(1): 49-56

5. Borrell A, Canto G, Pastor T, Aguillar, A. Organochlorine compounds in common dolphins (Delphins delphis) from the Atlantics and Mediterranean Water of Spain. Environ Pollut 2001;114: 265-74.

6. Storelli M, Marcotrigiano, G. Levels and congener pattern of polychlorinated biphenyls in blubber of the Mediterranean bottlenose dolphins Tursiops truncates. Environ Int 2003; 28:559-65.

7. Baird, C. Química ambiental. España, Editorial: Reverté, 2001: 313-20

8. Mojica, JI; Calle V JC . Libro Rojo De Peces Dulceacuícolas De Colombia. Bogotá, Colombia: Instituto de Ciencias Naturales, 2002: 15-25.

9. Oyakawa OT. Checklist of the freshwater fishes of South and Central America. Porto Alegre Brazil, Editora da Pontifícia Universidade Católica do Rio Grande do Sul; 2003: 238-240
10. Galvis G, Mojica JI, Camargo M. Peces del Catatumbo. Bogotá D.C: Asociación Cravo Norte; 1997: 25-30.

11. Tordecilla PG, Sánchez BS, Olaya NC. Crecimiento y mortalidad del Moncholo (Hoplias malabaricus, Bloch, 1794) en la cuenca del río Sinú, Colombia. Rev MVZ Córdoba 2003;8:(1): 278-284.

12. Kannan K, Tanabe S, Ramesh A, Subramanian A, Tatsukawua R. Persistent organochlorine residues in foodstuffs from Indian and their implications on human dietary exposure. JAFC 1992; 40:518-29.

13. Giesy J, Kannan K. Dioxin-like and non-dioxin-like toxic efects of polychlorinated biphenyl (PCBs): implications for risk assement critical. Rev Toxicol 1998; 28:511-69.

14. Argumedo C; Uparela, P. Validación e implementación de un método para determinación de pesticidas organoclorados en aguas, peces y sedimentos mediante la técnica de cromatografía de gases con detector captura de electrones. Facultad de Ciencias Básicas e Ingenierías, Departamento de Química, Universidad de Córdoba, Montería, Colombia 2007.

15. Miller JN, Miller JC. Estadística y Quimiometría para Química Analítica. 4 Ed. Madrid: Pearson Educación S.A. 2002: 143-152.

16. Plata J, Campos N, Ramírez G. Flujo de compuestos organoclorados en sedimentos de la Ciénaga Grande de Santa Marta. Caldasia 1993; 17(2):199-204.

17. Gneri F S, Angelescu V. La nutrición de los peces iliófagos en relación con el metabolismo general del ambiente acuático. Rev Inst Nac Invest C N Cienc Zool 1951;2(1):1-4. 
18. Lledos J, Morell M, Sánchez J. Distribución de los microcontaminantes orgánicos en un ecosistema fluvial. Significación de los estudios de impacto. Fish Res Board Can 1992; 25 (2443):65-70.

19. Voorspoels, S. et al. Levels and profiles of PCBs and OCPs in marine benthic species from the Belgian North Sea and the Western Scheldt Estuary. Mar Pollut Bull 2004;49: 391.

20. Reinert $\mathrm{R}$, Bergman $\mathrm{H}$. Residues of DDT in Lake trout(Salvelinus namaycush) ans coho salmon(Oncorhynchus Kisutch) from the Great Lake. Res Board Can1974; 31 (191):220-32.

21. Monod G, Keck G. PCBs in Lake Geneva (Lake Leman) fish. Bull Environ Contam Toxicol 1982; 29(55):570-6.

22. Giouranovits-Psyllidou $\mathrm{R}$, Georgakopoulos-Gregoriades E.Vassilopoulou V. Monitoring of organochlorine residues in red mullet (Mullus barbatus) from Greek Water. Bull Environ Contam Toxicol $1994 ; 28(22): 121-3$.

23. Allison D, Kallman B, Cope B, Valin C. Insecticide: effects on cutthroat trout of repeated exposure to DDT. Science 1963;142(3594):958-61.

24. Kellogg R, Bulkley R. Seasonal concentrations of dieldrin in water channel catfish, and cattfishfood organisms Des Moines River Iowa 1971/1973. Pestic Mont J $1976 ; 9(4): 86-94$.
25. Mora M, Papoulias D, Nava I, Bucker D. Comparative assesment of contaminants in fish from four resacas of the Texas USA-Tamaulipas, Mexico border region. Environ Int 2001;27:15-20.

26. Dybern BI, Dalziel J, Baker C, Jensen $S$. Manual de métodos de investigación del medio ambiente acuático. Análisis de presencia de metales y organoclorados en los peces. Roma: FAO; 1983.

27. Betancourt J, Ramirez G. Study of the processes related to the presence of organochloride pesticides in the Ciénaga Grande de Santa Marta. Bol Invemar 2005;34(1):121-39.

28. Samiullah Y. Biological monitoring of environmental contaminants. London: University of London; 1990.

29. Kalyoncu L; Agca I And Aktumsek A. Some organochlorine pesticide residues in fish species in Konya, Turkey Chemosphere. 2009;74: 885889.

30. Muralidharan Subramaniyan; Dhananjayan Venugopal And Jayanthi Palaniyappan. Organochlorine pesticides in commercial marine fishes of Coimbatore, India and their suitability for human consumption Environ Res 2009; 109: 15-21. 